\title{
¿Qué se aprende sobre la enseñanza en la carrera de formación de profesores? El caso Profesorado en Comunicación Social de la Universidad Nacional de Córdoba, Argentina
}

\author{
What is learned about teaching in the career of teacher training? The case \\ in Faculty of Social Communication of the National University of Cordoba, \\ Argentina
}

\author{
María Alejandra Salgueiro \\ Magíster en Administración Educativa \\ Universidad Nacional de Córdoba, Argentina \\ Correo electrónico: asalguei@live.com.ar
}

Recibido: 12 de febrero de 2016; aprobado: 1 de abril de 2016

\section{Cómo citar este artículo:}

Salgueiro, M.A. (2016) ¿Qué se aprende sobre la enseñanza en la carrera de formación de profesores? El caso profesorado en Comunicación Social de la Universidad Nacional de Córdoba, Argentina. Espiral, Revista de Docencia e Investigación, 6(1), $137-148$.

\section{Resumen}

Tipología y objetivo. El presente artículo de investigación comparte los resultados alcanzados hasta el momento de una investigación titulada "La construcción de conocimientos sobre la enseñanza en el marco de las carreras de formación docente destinadas a graduados universitarios" que dirijo desde la Cátedra Práctica Docente y Residencia, del Profesorado de Comunicación Social de la Universidad Nacional de Córdoba, Argentina, en calidad de profesora adjunta a cargo de esta cátedra.

Metodología. Dicho estudio en esta primera etapa tuvo la intención de identificar y analizar los conocimientos-saberes (aprendizajes) que se construyen durante la carrera del Profesorado vinculados a la enseñanza en general y a la enseñanza de un campo de conocimiento particular, como lo es el de la comunicación social. (sí son los objetivos del estudio)solicito incluir en metodología: El trabajo se inscribe en el marco de los enfoques cualitativo-interpretativos. Se llevó a cabo un trabajo de campo que consistió en una primera etapa en el relevamiento de las percepciones y opiniones de los egresados de la carrera acerca de su formación. Se utilizaron dos técnicas: cuestionarios con preguntas abiertas y entrevistas en profundidad.

Resultados. Los resultados alcanzados dan cuenta de algunas vacancias y debilidades en relación con la carrera de profesorado, reflexión que contribuye sin duda al campo de la pedagogía y la didáctica de la formación.

\section{Palabras claves}

Formación docente, enseñanza, conocimientos, comunicación social.

\begin{abstract}
This research article shares the results achieved for an investigation entitled "Building knowledge about teaching in the context of teacher training courses addressed to university graduates". This research is done as part of the subject "Practice Teaching and residence" of faculty of Social Communication of the National University of Córdoba-Argentina, as an Adjunct Professor in charge of this subject.

This study at this early stage intended to identify and analyze the knowledge (learning) built during the career of teachers related to teaching in general and teaching a particular field of knowledge, as Social Communication.

The results show some vacancies and weaknesses related to the career of teachers, a reflection that undoubtedly contributes to the field of pedagogy and didactics education.
\end{abstract}

\section{Keywords}

Teacher training, education, skills, social communication. 


\section{Introducción}

La docencia es una actividad profesional que requiere una formación específica orientada al desempeño de un rol, de un trabajo, el trabajo de enseñanza, su dimensión sustantiva. Entre los contenidos que estructuran la formación de un docente la enseñanza ocupa un lugar destacado y que las personas que transitan estas carreras han construido nociones, conocimientos, aprendizajes en relación con esta noción. Sin embargo, quienes somos responsables de los procesos de formación notamos que no resulta sencillo precisar qué es lo que han aprendido quienes finalizan estas carreras respecto de la enseñanza en su doble dimensión, conceptual y práctica. Este interrogante se actualiza sobre todo en los espacios de evaluación de las cátedras y todavía más, en las instancias de las prácticas y residencia.

Nuestro interés como equipo de cátedra y en tanto formadores, es contribuir en esta precisión. Las preguntas que orientaron esta búsqueda fueron: ¿Qué saberes implicados en el oficio, trabajo de profesor, referidos a la enseñanza y en especial a la enseñanza de la comunicación, los egresados reconocen construir durante la carrera? Algunos indicios nos los están señalando los egresados de la carrera que hemos optado como unidad de análisis, a partir de la aplicación de cuestionarios y la realización de entrevistas en profundidad los que pretenden reconstruir su experiencia formativa en el marco de la carrera.

El presente trabajo hace referencia a los resultados obtenidos hasta este momento, los cuales nos dan luz sobre la problemática objeto de estudio, y a repensar las propuestas de enseñanza en el profesorado.

\section{Datos de contexto: El Profesorado universitario de Comunicación Social}

En el 2011 la Facultad de Comunicación Social de la Universidad Nacional de Córdoba implementó la carrera de Profesorado en Comu- nicación Social, como un ciclo de complementación curricular para los que cuentan con la formación disciplinar y una titulación universitaria como comunicadores. Esta carrera da respuesta a una necesidad del sistema educativo: disponer de personal con formación pedagógica para asumir la enseñanza de los espacios curriculares vinculados a la comunicación. En este espacio de formación confluyen saberes académicos (de referencia) y conocimientos prácticos o prácticas profesionales correspondientes al campo "comunicación" y al campo de la pedagogía y didáctica. Los primeros adquiridos en la carrera de grado, los segundos por abordar e integrar en esta formación.

\section{Enseñanza y conocimientos}

Analizar los conocimientos que los egresados reconocen haber aprendido en la carrera acerca de la enseñanza requirió explorar la producción actual en torno a esta problemática. La pregunta que orientó esta indagación fue: ¿Qué conocimientos supone el trabajo de enseñanza?

Respuesta que se ata a la concepción de enseñanza que adscribimos en el estudio: una combinación inédita y una organización singular de las actividades a través de las cuales, un actor, en este caso el docente, interviene sobre la realidad, en el marco de una serie de sucesos en curso, con la intención de generar procesos formativos (Barbier y Galatanu, en Cols, 2011, p. 61). En tanto práctica social, se sostiene sobre procesos interactivos múltiples (Edelstein, 2005), responde a necesidades e intenciones que exceden lo individual y solo pueden entenderse en relación con un contexto histórico, social e institucional del que forman parte (Edelstein, 2011). Se reconoce además que posee cierto nivel de incertidumbre, imprevisión y ambigüedad, rasgos que brindan fundamento a la naturaleza "práctica" de la enseñanza (Schwab, 1973; Schön, 1992). 
Las decisiones que toman los docentes atañen a un aspecto central y constitutivo de su trabajo: el problema del conocimiento, cómo se comparte y construye en los espacios educativos (Edelstein, 2011).

Enseñar involucra procesos múltiples de interacción y mediación entre sujetos y los conocimientos con la intención de que los sujetos en formación se apropien, construyan conocimientos de relevancia social.

Por lo tanto, la enseñanza es interactiva e intencional, está ligada a la transmisión de saberes, tarea que integra asimismo aspectos organizativos -ligados al sostén de un encuadre de trabajo- y relacionales que atañe al logro y mantenimiento de una relación pedagógica (Cols, 2011).

La enseñanza supone entonces incorporar construir conocimientos múltiples, de diferente naturaleza, provenientes de diversas fuentes-.

Existe cierto consenso en reconocer al menos dos tipos de conocimiento de los profesores: uno más formalizado compuesto de teorías, conceptos, procedimientos sistematizados y que cuentan con cierto estatus científico y legitimidad, y otro más práctico, compuesto por esquemas de pensamiento y decisión construidos en la experiencia, en la ambigüedad e incertidumbre de las prácticas (Edelstein, 2011).

Flavia Terigi (2012) identifica como saber específico y particular de los docentes el saber sobre la transmisión. Dicho saber solo se produce en relación con un campo de conocimiento determinado, en consecuencia, los docentes son expertos en un doble sentido:

como expertos en un/os campo/s cultural/ es y como expertos en las intervenciones pedagógicas que se requieren para que grupos de alumnos puedan avanzar en su dominio de los saberes propios de esos campos. Esta doble especialidad del docente implica una relación peculiar con el conocimiento producido fuera de la escuela, que no es la del productor del saber a transmitir, sino la de quien opera la transmisión cultural (Terigi, 2012, p. 10).

El saber sobre la transmisión se vincula con la categoría "conocimiento pedagógico del contenido" propuesta por Lee Schulman en el marco de un programa de investigación orientado a estudiar el desarrollo del conocimiento del docente en la enseñanza. Esta categoría involucra

los saberes que le permiten a un docente hacer enseñable un contenido. Implica la comprensión cognitiva del contenido de la enseñanza lo cual lo habilita para comprender qué dificultades puede presentar un tópico en su aprendizaje, qué concepciones e ideas previas tienen los estudiantes sobre el mismo y qué formas de representación posibilitan su comprensión (Shulman, 1986 en Salazar, 2005).

Representa la mezcla entre materia y didáctica por la que se llega a una comprensión de cómo determinados temas y problemas se organizan, se representan y se adaptan a los diversos intereses y capacidades de los alumnos, y se exponen para su enseñanza (Shulman, 2005, p. 11).

La construcción de conocimiento pedagógico del contenido no es una operación sencilla. Una de las razones tiene que ver con que los contenidos de enseñanza se nutren de conocimientos producidos en ámbitos no escolares, dichos conocimientos no tienen la intencionalidad, al menos en principio, de ser enseñados en el marco de los sistemas educativos. La decisión de que algo de la producción académica forme parte de la enseñanza exige operar "didácticamente" sobre ese conocimiento, lo que implica entre otras cuestiones, ajustes, contextualizaciones, adaptaciones, traducciones. Al respecto Gloria EdesItein propone la categoría "construcción metodológica" (Edelstein, 2011, p. 147) para referirse a este trabajo didáctico que es necesario realizar para que un conoci- 
miento sea objeto de enseñanza y aprendizaje, lo que exige repensar la relación entre conocimiento, modos de transmisión, los sujetos y las organizaciones. Supone reconocer que los contextos de producción del conocimiento no son los mismos que los contextos de transmisión, de enseñanza. La enseñanza entonces articula sustancias y formas, contenidos y modos de trabajo adecuados para garantizar el aprendizaje de todas las personas.

Veremos en los resultados que este conocimiento no es del todo reconocido o destacado.

\section{Aspectos metodológicos}

El estudio consta de dos etapas, la primera tiene la intención de identificar y analizar los conocimientos-saberes (aprendizajes) que se construyen durante la carrera de Profesorado, vinculados a la enseñanza en general y a la enseñanza de un campo de conocimiento particular, como lo es el de comunicación social. La segunda, pretende analizar la relación entre la construcción de dichos conocimientos y saberes y las propuestas de cátedra.

La identificación y análisis de los conocimientos aprendidos se llevó a cabo desde la perspectiva de los principales actores implicados, los egresados de la carrera. Las técnicas de recolección de información consistieron en:

a) Cuestionarios con preguntas abiertas que indagaron acerca de qué esperaban aprender en el profesorado sobre la enseñanza, cuáles de estos conocimientos fueron abordados, qué es necesario saber para enseñar comunicación, qué aprendieron sobre la enseñanza, en especial la enseñanza de la comunicación. Las respuestas de los cuestionarios permitieron un primer nivel de análisis de carácter descriptivo.

b) Entrevistas en profundidad a los egresados de la carrera a los efectos de ampliar y profundizar las respuestas ofrecidas en los cuestionarios y narración de una experiencia personal en la que hayan observado/participado de una clase -en el marco del Profesorado (como alumno, observador de un par o practicante) que, desde su punto de vista, haya favorecido/ propiciado aprendizajes sobre la enseñanza. Tanto las entrevistas como las narrativas posibilitaron arribar a un segundo nivel de análisis más de orden interpretativo.

\section{Proceso de análisis}

Con el fin de orientar el análisis se elaboró una matriz que consta de dos dimensiones:

La enseñanza desde una perspectiva general, refiere a: los conocimientos teóricos y conceptualizaciones (saberes de referencia), los esquemas de decisión y acción (saberes prácticos).

La enseñanza de un contenido específico, la cual integra dos subdimensiones:

- el saber sobre el campo de conocimiento particular.

- el saber sobre su transmisión-enseñanza.

\section{Resultados}

Se presenta una síntesis de los resultados alcanzados, organizados en torno a los principales tópicos indagados en el trabajo de campo y que brindan conocimiento sobre el objeto de estudio.

Lo que se espera aprender en el Profesorado

Las respuestas oscilan entre los dos polos de toda formación académico-profesional: los conocimientos de orden teórico y las herramientas prácticas. Algunos refieren a la necesidad de contar con marcos teóricos que brinden fundamento pedagógico a las decisiones de la docencia que vienen ejerciendo de modo intuitivo. Es necesario aclarar que al momento de iniciar la carrera, un porcentaje significativo de los alumnos se desempeñaban como profesores en el sistema educativo obligatorio, a pesar de no contar con titulación docente. 


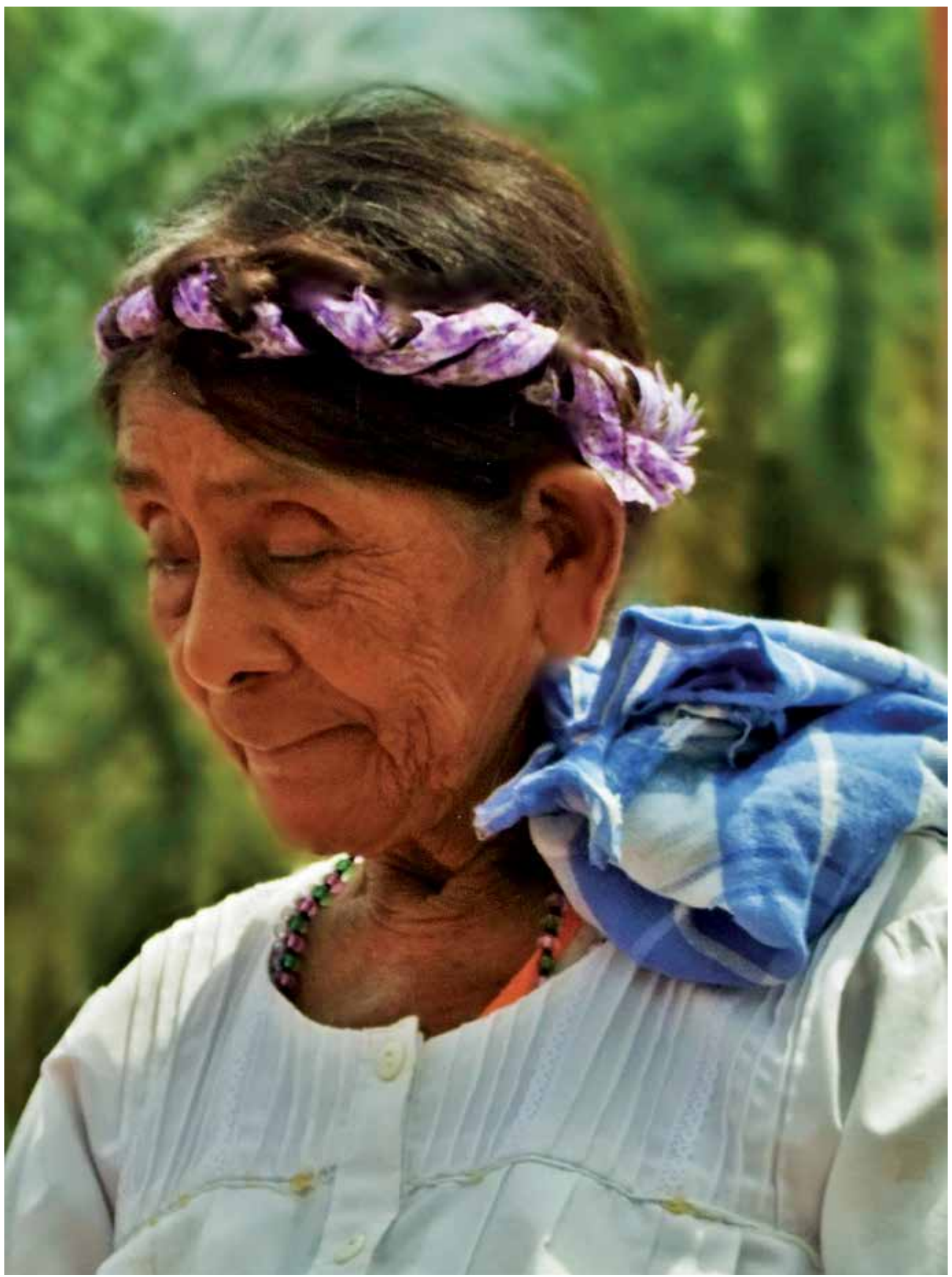


La alusión a estos marcos es muy general y poco precisa, se habla de conocimientos de pedagogía, el acceso a textos pedagógicos, el rol docente y los vínculos con los estudiantes y sus aprendizajes.

Respecto de las herramientas prácticas, se desprende una sobrevaloración de las mismas al punto de reducir la enseñanza al manejo de buenas técnicas y herramientas, perspectiva que ha impregnado la formación de docentes años atrás y que aún persiste sobre todo en aquellos que deciden realizar una formación pedagógica complementaria a su formación disciplinar. Se percibe una representación de la pedagogía y la didáctica muy rudimentaria consistente en "brindar técnicas para enseñar lo que ya sé" (Núñez y Cubillos, 2012, p. 87) que garanticen una buena clase y sin "contratiempos".

\section{Así lo expresan:}

Esperaba que se me presentaran textos pedagógicos a los que no había accedido en la carrera de grado y que me fueran útiles para discutir (conmigo y mis colegas) la práctica docente que estaba/estábamos desarrollando.

Herramientas pedagógicas prácticas y concretas para plasmar la teoría adquirida en la carrera de grado, en el espacio educativo que me tocara trabajar.

Esperaba encontrar recursos teóricos porque en mi trabajo hasta ahora me manejé en el aula en la prueba, ensayo y error... con la teoría y los autores te da el marco con lo que te legitima tu quehacer docente y potencia, te da más seguridad.

Lo que esperaba aprender es poder armar una buena clase; que el profesorado me diera las herramientas pero en un sentido muy práctico, es decir, yo esperaba "la receta" el poder despuntar el $A B C$ de cómo se construye una buena clase. Esperaba el conocimiento de las herramientas pedagógicas y didácticas que me permitieran desplazarme y transitar una clase sin mayores contratiempos...
Algunas respuestas aluden a la dimensión relacional, a contar con aquello que es necesario para trabajar con los alumnos y construir un buen vínculo:

Saberes en relación a trabajar con las potencialidades del alumno y no desde los puntos débiles.

Esperaba aprender a manejarme en un aula, a tener más herramientas con respecto a manejo de grupos, aprender estrategias que fomentaran el vínculo pedagógico...

\section{Lo que se necesita saber para enseñar}

La mayoría de las respuestas refuerzan los aspectos didácticos y relacionales involucrados en la enseñanza. Resultan enunciados muy generales no exclusivos de la formación de un profesor de comunicación:

Pedagógicos y didácticos, otros vinculados a la lectura e interpretación del curriculum (su relación con las planificaciones, programas, PEI y $\mathrm{PCI}$ ), las secuencias didácticas, las TIC, la evaluación. No son exclusivos de un profesor en Comunicación, pero son los primeros que se me vienen a la mente...

Para trabajar como docente es necesario el vínculo... El encuentro con otro es un riesgo, es necesario comprender que depende del otro el acuerdo por más que uno haga un montón (es la opinión de un egresado).

Algunos entrevistados se aproximan al saber sobre la transmisión y la mediación que es necesario producir para facilitar la construcción de conocimientos y cuáles son sus condiciones:

Un saber simplificar, darle vida a la teoría, saber hacerlo, un vínculo con el conocimiento para poder transmitir...

Saber traducir una teoría o concepto para su efectiva apropiación...

Muy pocos hablan de los conocimientos disciplinares, aquellos que son propios del campo de la comunicación, estos no son planteados desde una perspectiva didáctica, es decir, en 
tanto contenidos de enseñanza y de aprendizaje en el marco de un proyecto educativo:

El análisis de los medios, las tic, las teorías de la comunicación, comunicación y cultura.

Actualizar o reactualizar lo que hemos estudiado en comunicación, más que nada los que somos más viejos... (respuesta-opinión de un egresado).

Lo que se aprende en la carrera sobre la enseñanza, la enseñanza de la comunicación (respuesta-opinión de un egresado).

Replican las respuestas ofrecidas en relación con sus expectativas sobre lo que suponen van a aprender en la carrera, aparecen referencias sobre cuestiones teóricas que son muy generales e indefinidas y cuestiones más ligadas al trabajo pedagógico en sus diferentes fases preactiva y activa (planificación y desarrollo de la clase) y algunas pocas relativas a la inscripción institucional y organizativa de la enseñanza. Aparecen en casi todas las respuestas referencias sobre la cuestión vincular.

Algunos apuntan sobre la relación entre la teoría y la práctica planteada de modo muy vago, ya que no se precisa en qué sentido la teoría fundamenta la práctica o qué aporta la teoría al trabajo de enseñanza. Cuando se habla de los aspectos prácticos no aparece su vinculación con la teoría o con algún tipo de proceso reflexivo, podría inferirse que la práctica refiere solo a lo instrumental. Sí se advierte la diferencia o "distancia" entre los contextos de producción académica y "los escenarios reales".

Algunas nociones, conceptos y marcos teóricos propios de la pedagogía que son necesarios para la enseñanza:

Aprendí aspectos teóricos y conocimientos de profesores y muchos de los saberes de colegas quienes me iban haciendo el encuadre de la distancia entre lo académico y los escenarios reales... respuesta-(opinión de un egresado). ...el armado del programa hasta el momento de la clase y los materiales y la manera de dictarla (respuesta-opinión de un egresado).

Los conocimientos sobre el curriculum (incluyendo el oculto), la planificación y secuencia de las clases, la autoevaluación o reflexión sobre la práctica, la evaluación. Como experiencias, la observación y análisis de otras planificaciones y clases de colegas

que el currículo funciona como un vademécum de temas, está listado, fragmentado y descontextualizado, analizamos la posibilidad de ver los temas como una red de relaciones hablamos de la importancia del vínculo pedagógico a veces como posibilitador y otras como condición necesaria (respuesta-opinión de un egresado).

Respecto de la dimensión institucional, se señala:

...me hizo despertar la mirada en todo lo que implica la intervención áulica/escolar/institucional.

...El profesorado me ofreció seguridad y confianza, herramientas que me fortalece, como la capacidad para analizar la realidad institucional, ver el contexto, la diferencia entre las instituciones, las distintas realidades, observar, mirar, estar atento y alerta, no ponernos el casete como si todo fuera lo mismo (respuesta-opinión de un egresado).

Varios son los que resaltan la dimensión político-ética de la enseñanza, reconocen como temática muy trabajada en el profesorado el posicionamiento del docente en relación con el conocimiento, con el aprendizaje y con los estudiantes. Algunos enfatizan aspectos de orden emocional y afectivo, una perspectiva humana y subjetiva de la enseñanza.

...rescatar la importancia del posicionamiento como sujeto del conocimiento y de las posibilidades que sí tienen los estudiantes de completar, complementar. Del rol activo por parte del educando y el educador y de la común unión de ambos... 
la relación pedagógica, la confianza que es condición para enseñar

...fundamentalmente re encontrar en la tarea una mayor motivación

...aprendí durante las prácticas a cómo manejar la clase, enganchar a los estudiantes y entusiasmarlos...

reconocerme como sujeto de enseñanza, de aprendizaje quien es responsable de construir un vínculo con sus estudiantes y acompañarlos en sus proceso de aprendizaje.

El docente es un guía, no tiene que mostrarse como el único que sabe. Yo pude trasladar mi experiencia en educación no formal a la experiencia de aula en las prácticas, la carrera me reforzó esta perspectiva.

...lo que pude hacer durante la formación es desenclavar este concepto -enseñanza- de sus componentes más subjetivos y experienciales y "disecarlo" verlo en su complejidad todo lo que implica e involucra el enseñar, el pararse a enseñar: aspectos estratégicos, metodológicos, didácticos pedagógicos, y también políticos y psicológicos... (respuesta-opinión de un egresado).

Pocos mencionan haber aprendido algo en relación con la especificidad de la enseñanza de comunicación:

...nos dio herramientas didácticas específicas de la carrera del campo disciplinar considerando que la didáctica de la comunicación es un campo todavía en construcción.

tener en cuenta la perspectiva de los estudiantes, los procesos de aprendizaje, algunas estrategias...

En algunas respuestas se proponen títulos contenidos como extraídos de un programa de cátedra, otros realizan una traducción y lo vinculan con el campo de trabajo-:

los distintos modos de enseñanza a lo largo de la historia de la educación...
Las teorías del aprendizaje tan importante para entender estos procesos

...modelos de enseñanza propuestos por los profesores de la carrera que hacen reflexionar y preguntarse por la razón y sentido como lo fue en los espacios de las prácticas.

formas diferentes de enseñar, más hacia el constructivismo, el docente más como guía que como quien porta el saber...

Algunos cuestionan el valor e incidencia de los conocimientos teóricos en su formación, son varios los que reconocen la fuerza de los relatos y experiencias de sus compañeros que se desempeñan como profesores en la construcción o replanteo de ideas sobre la enseñanza. Si bien el saber de la experiencia (Contreras, 2010) es valioso, ya que ofrece modos de intervención posibles y respuestas pertinentes a los problemas de la práctica, aparece como un contenido aleatorio o casual, contrapuesto al ofrecido por las cátedras. Es decir, no se percibe un trabajo de integración entre marcos teóricos y prácticas que permita comprender y resignificar ambos.

...hubo un replanteo de ideas, revolucionadas más por las experiencias de otros que por las teorías del profesorado... El tránsito por el profesorado fue, mas bien, una exposición de realidades ajenas y/o compartidas que sirven a la práctica, más no la revolucionan, como si lo hace el ejercicio de la docencia.

...pienso que fue muy rico observar a los colegas dando clases, aprendí quizá más que leyendo teoría...

Conceptos-significados en torno a la enseñanza

De las tres instancias de las entrevistas se desprenden ideas y concepciones sobre la enseñanza que resulta un insumo interesante para repensar la enseñanza de esta noción en el marco del profesorado. 


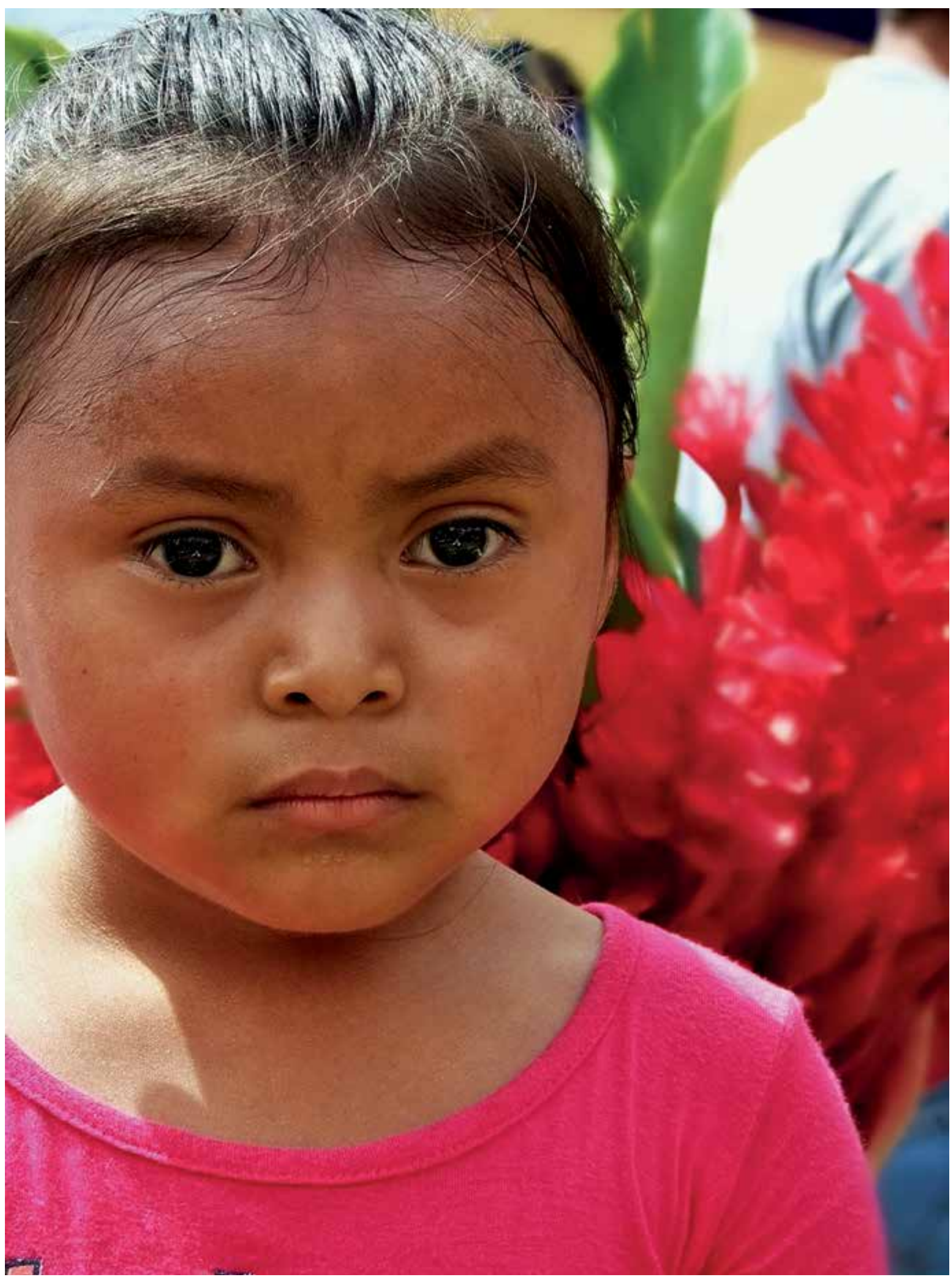


Se percibe una conceptualización básica, imprecisa y en algunos casos precarios de la enseñanza, para algunos se equipara a la comunicación sin ofrecer ningún tipo de distinción o especificidad entre una y otra. Las siguientes citas lo ilustran:

Enseñar es compartir, comunicar y construir, si me preguntan qué es comunicar, pues es lo mismo. Se incluyen y conviven. En este momento trabajo en los dos ámbitos y no considero que difieran, solo lo harían si tenemos en cuenta las instituciones establecidas.

compartir, comunicar, común unión, entrega, feed back, reconocimiento de otro, respeto y esperanza de que con el conocimiento se es más libre...

es elegir la mejor forma de comunicar ideas, de emancipar al otro...

establecer una comunicación en la que se comparte algo que uno tiene y que el otro no o cree no tenerlo y al mismo tiempo estar abierto a repensarlo...

Otros la equiparan con la educación y la formación:

Para mí enseñar es dejar huella y ¿qué es dejar huella?, es aquello que perdura pero no solo porque estuvo bien enseñado - explicado sino porque en alguna medida quien lo transmitió tuvo que saltar de un molde para hacerlo así...

Algunos se aproximan a los componentes de todo acto de enseñanza, el conocimiento y los sujetos aunque expresados vagamente:

...brindar al otro algún tipo de conocimiento, no necesariamente tiene que ver con algo teórico, puede ser algo vivencial... (respuesta-opinión de un egresado)

En relación con las tres dimensiones definidas en la matriz de análisis, se advierte una ausencia de referencias sobre el conocimiento que es necesario construir para enseñar comunicación en los contextos educativos en los que los egresados podrán desempe- ñarse. Esta última se la menciona como significado y práctica pero no tanto como contenido de enseñanza. No se señala como conocimiento particular, singular del docente el saber sobre la transmisión (Terigi, 2012), el conocimiento pedagógico del contenido (Shulman, 2005) correspondiente al campo de su especialidad, tampoco perciben que se haya aprendido algo sobre esto, a pesar de que la carrera cuenta con espacios en los que estas temáticas serían abordadas.

Por lo tanto, se enfatizan los aspectos generales de la enseñanza destacándose entre ellos los relativos a las herramientas didácticas y cuestiones prácticas, sin ahondar en lo que estas implican. En sus expectativas plantean una suerte de "traducción directa de las piezas de conocimiento declarativo o procedimental impartidas en cursos de acción práctica" (Terigi, 2011, p. 51) que podría reflejar una concepción simplificada de la enseñanza. Esta perspectiva general excluye el saber didáctico propio de los niveles y modalidades educativas del sistema educativo -sus sujetos y contextos- para los cuales la carrera forma.

Algunos señalan la faceta relacional de la enseñanza, la necesidad de saber construir buenos vínculos con los estudiantes. Pocos aluden a la dimensión política e institucional, a las prescripciones curriculares que dan marco a la enseñanza. Las conceptualizaciones que se ofrecen son muy vagas y generales, equiparando la enseñanza a la comunicación.

\section{Conclusiones}

Los resultados alcanzados son hasta ahora un indicio de lo que se logra aprender en la carrera, cuestión que posibilita repensar la formación que brinda el Profesorado de Comunicación Social.

Podría hablarse de vacancias, ausencias de contenidos de la formación y de ciertos modos de abordaje que no estarían posibilitando la construcción de saberes de referencia claros y 
potentes que a su vez den lugar a la reflexión sobre el objeto de trabajo de un profesor y su complejidad, no reducido a la aplicación de técnicas o al uso de herramientas didácticas, como tampoco a la producción de buenos vínculos con los estudiantes. Retomamos la idea de vacancia que Flavia Terigi emplea al analizar la formación de docentes secundarios en Argentina, quien la entiende como

lugar que se percibe como libre y que puede ser ocupado, en este caso por conocimientos pertinentes; tiene además la virtud de permitir que se exprese que un lugar vacante no necesariamente está vacío, sino que puede haber sido ocupado por otra cosa... (Terigi, 2011: 14).

Una vacancia o temática por profundizar es la especificidad de la enseñanza en relación con la comunicación desde el punto de vista conceptual y en tanto práctica profesional. Si bien se reconoce que la enseñanza y la comunicación son prácticas afines y convergentes, a su vez resulta importante reconocer que cada una posee objetos de estudio, producciones y recorridos distintos. En los relatos se mencionan de forma indistinta como si fueran lo mismo. Preocupa esta indiferencia en un egresado de comunicación social que ha finalizado una formación pedagógica y didáctica.

Otra cuestión que alerta sobre la formación de la carrera tiene que ver con la enseñanza de los contenidos del campo de la comunicación, temática que compromete a la didáctica específica. Aunque se admite que este campo de conocimiento está en proceso de conformación, es nuevo en la historia de las didácticas especiales, no deja de llamar la atención que los entrevistados no mencionen que hayan aprendido algo sobre esta cuestión, que hayan construido algún tipo de saber ligado a la enseñanza de los contenidos propios del área o disciplina de su especialización, saber que se supone debería ser el eje de la formación.

Se infiere un cuestionamiento al lugar y valor de la teoría en la formación, si bien varios plan- tean la necesidad de contar con fundamentos teóricos provenientes de la pedagogía, estos no parecen colmar sus expectativas, pareciera que no han cumplido con su cometido.

Las expectativas no cumplidas del todo con lo teórico es probable que guarden relación con una visión aplicacionista y deductiva de la teoría a la práctica, que puede desprenderse de algunos relatos, por cuanto se espera que la teoría casi en automático ofrezca las respuestas a las situaciones de la práctica.

Asimismo, el valor incierto de las teorías y una didáctica no del todo bien posicionada en esta carrera podría explicar las referencias ambiguas, imprecisas, indefinidas en relación con la enseñanza, no aparecen nombradas sus dimensiones, fases, componentes, determinaciones, entre otros aspectos. Es de destacar que no se refieran -o solo lo planteen unos pocos- a la tríada didáctica, a los contenidos de enseñanza, su relación con las prescripciones curriculares, las cuestiones organizativas y contextuales de toda práctica de enseñanza. No se la reconoce como una acción intencional, interactiva, institucional, en el marco de una serie de sucesos en curso. Tampoco se destaca uno de los aspectos más significativos de la enseñanza, que "las decisiones que toman los docentes atañen a un aspecto central y constitutivo de su trabajo: el problema del conocimiento, cómo se comparte y construye en los espacios educativos" (Edelstein, 2011).

Sí aparece en la mayoría de los relatos y respuestas la necesidad de contar con herramientas didácticas, las cuales no estan señaladas en lo que ellos identifican como conocimientos aprendidos en la carrera.

Los resultados alcanzados hasta el momento inquietan, provocan nuevos interrogantes que será necesario responde en el transcurso de la segunda etapa de este estudio. Se asume que la investigación educativa tiene la responsabilidad de producir cambios y transformacio- 
nes, en este caso, la revisión y modificación de las propuestas de enseñanza de las carreras de profesorado. Es probable que el desarrollo de este estudio lo esté propiciando así como la generación de insumos para profundizar el conocimiento sobre la pedagogía de la formación.

\section{Referencias}

Barbier, J.-M., Galatanu, O. (Dir.). (2004). Les savoirs d'action: une mise en mots des compétences? Paris: L'Harmattan.

Cols, E. (2011). Estilos de enseñanza. Rosario: Homo Sapiens.

Contreras, J. (2010). Pedagogías de la experiencia y la experiencia de la pedagogía. En Contreras, J., y Pérez, N. de. (Comps.). Investigar la experiencia educativa. Madrid: Morata.

Edelstein, G. (2011). Forma y formarse en la enseñanza. Buenos Aires: Paidós.

Núñez, M., y Cubillos, L. (2012). Saber disciplinario y saber pedagógico: tensiones y respiros en un programa de formación inicial docente consecutivo. Revista Docencia, 47, Santiago de Chile. Recuperado de http://www. revistadocencia.cl/pdf/20120920232204.pdf
Salazar, S. (2005). El conocimiento pedagógico del contenido como categoría de estudio de la formación docente. Instituto de Investigación en Educación. Universidad de Costa Rica. Recuperado de www.redalyc.org/articulo. oa?id $=44750211$

Shulman, L. (2005). Conocimiento y enseñanza: fundamentos de la nueva reforma. Revista de Currículum y Formación del Profesorado, 9(2). Recuperado de http://www. ugr.es/local/recfpro/Rev92ART1.pdf

Schwab, J. (1974). Un enfoque práctico para la planificación del currículo. Buenos Aires: El ateneo.

Terigi, F. (2009). La formación inicial de profesores de educación secundaria: necesidades de mejora, reconocimiento de sus límites. Revista de Educación, 350. Universidad de Buenos Aires y Universidad Nacional de General Sarmiento. Buenos Aires, Argentina. Recuperado de http:// www.revistaeducacion.educacion.es/re350/re350_06. pdf

Terigi, F. (2011) Aportes pedagógicos a la reformulación de la formación inicial de los/as profesores/as de escuela secundaria en Argentina. Informe Final. Ministerio de Educación, Buenos Aires. 